\title{
Point: Minimally invasive bipolar radiofrequency ablation of lone atrial fibrillation: Early multicenter results
}

\author{
Erik Beyer, MD, ${ }^{\mathrm{a}}$ Richard Lee, MD, ${ }^{\mathrm{b}}$ and Buu-Khanh Lam, $\mathrm{MD}^{\mathrm{c}}$
}

Objective: The treatment of lone atrial fibrillation can be a minimally invasive procedure using bipolar radiofrequency ablation technologies. Our objectives were to report on the safety and early efficacy of this novel therapeutic modality.

\begin{abstract}
Methods: At 3 North American institutions between February 2005 and August 2007, 100 patients underwent minimally invasive bilateral pulmonary vein isolation, autonomic denervation, and left atrial appendage resection. The mean age was $65 \pm 11$ years, and 70\% were male. The median duration of atrial fibrillation was 5.0 years; atrial fibrillation was paroxysmal in 39 patients $(39 \%)$, persistent in 29 patients $(29 \%)$, and permanent in 32 patients $(32 \%)$. Indications for surgery included failure of medical therapy or percutaneous ablation and severe symptoms. Mean follow-up was $13.6 \pm 8.2$ months.

Results: The mean operative time was $253 \pm 65$ minutes, and the median hospital length of stay was 5 days. There were no intraoperative conversions and no mortality to report. Postoperative complications included pacemaker requirement in 5 patients $(5 \%)$, phrenic nerve palsy in 3 patients $(3 \%)$, hemothorax in 3 patients $(3 \%)$, transient ischemic attack in 1 patient $(1 \%)$, and pulmonary embolism in 1 patient (1\%). At follow-up, 87\% of patients were in normal sinus rhythm (paroxysmal 93\%, persistent 96\%, permanent $71 \% ; P<.05$ ); antiarrhythmic therapy was discontinued in $62 \%$ of patients, and anticoagulation therapy was discontinued in $65 \%$ of patients.
\end{abstract}

Conclusion: Minimally invasive bipolar radiofrequency ablation of lone atrial fibrillation is a safe and efficacious therapeutic option in selected patients. Further development is needed to reduce the rate of complications. Longterm prospective results are required to further validate this modality as a therapeutic option to treat lone atrial fibrillation.

As many as 6 million Americans already have atrial fibrillation (AF). ${ }^{1}$ By 2020, AF could affect as many as 9 million people in the United States alone. Despite advances in medical therapy, medications have only been effective in restoring sinus rhythm (SR) in approximately one half of all the patients with $\mathrm{AF}^{2,3}$ This lack of successful SR restoration has limited the advantage of rhythm control in several large studies. ${ }^{2,4}$ However, AF remains a major cause of stroke and an independent risk factor for mortality. ${ }^{2,5,6}$ There is speculation that improved success in SR restoration may offer measurable survival advantages to the population with $\mathrm{AF}{ }^{2}$

Maze surgery was established as a curative procedure for patients with AF more than 2 decades ago and has been suggested to reduce stroke in patients with AF. ${ }^{7,8}$ Although more intensive monitoring suggests that results may be slightly less curative than historical reports state, ${ }^{9}$ the results

From the Scott and White Clinic, ${ }^{\mathrm{a}}$ Temple, Texas; Northwestern University, ${ }^{\mathrm{b}}$ Chicago, Illinois; and University of Ottawa Heart Institute, ${ }^{\mathrm{c}}$ Ottawa, Canada.

Presented at the 88th Annual Meeting of the American Association for Thoracic Surgery, May 11-14, 2008, San Diego, California.

Received for publication May 7, 2008; revisions received Oct 5, 2008; accepted for publication Nov 17, 2008.

Address for reprints: Buu-Khanh Lam, MD, Division of Cardiac Surgery, University of Ottawa Heart Institute, 40 Ruskin St, H3404, Ontario, Canada, K1Y 4W7 (E-mail: bklam@ottawaheart.ca).

J Thorac Cardiovasc Surg 2009;137:521-6

$0022-5223 / \$ 36.00$

Copyright $\subset 2009$ by The American Association for Thoracic Surgery

doi:10.1016/j.jtcvs.2008.11.031 of the complete Maze procedure are clearly more successful than catheter-based interventions. ${ }^{10}$ Unfortunately, the Maze procedure has not been embraced as a stand-alone procedure, with only 600 cases performed for lone AF in the Society of Thoracic Surgeons database in $2006 .^{11}$

New technologies have allowed the creation of transmural lesions on a beating heart through alternative, less-invasive incisions. Although many of these have demonstrated early success, ${ }^{12,13}$ the results are often not as good in larger series. ${ }^{14}$ One of these new technologies has been the application of bipolar radiofrequency ablation to create pulmonary vein isolation via a bilateral thoracotomy approach. ${ }^{15,16}$ However, the series are generally small and limited to a single institution.

The objective of this study was to examine the results of this approach in a multi-institutional setting. In addition to the pulmonary vein isolation, autonomic ganglionic plexi (AGP) stimulation and ablation and left atrial (LA) appendage ligation and resection were performed at all institutions.

\section{PATIENTS AND METHODS \\ Patient Population}

At 3 North American institutions between February 2005 and August 2007, 100 patients underwent thoracoscopic-assisted bilateral pulmonary vein isolation, autonomic denervation, and LA appendage resection via bilateral mini-thoracotomies. Main indications for surgery were failure of medical therapy or percutaneous ablation and severe symptoms. The 


$$
\begin{aligned}
& \text { Abbreviations and Acronyms } \\
& \text { AF }=\text { atrial fibrillation } \\
& \text { AGP }=\text { autonomic ganglionic plexi } \\
& \text { LA }=\text { left atrial } \\
& \text { NSR }=\text { normal sinus rhythm } \\
& \text { SR }=\text { sinus rhythm }
\end{aligned}
$$

mean age was $65 \pm 11$ years. Baseline patient characteristics are described in Table 1.

Mild aortic valvular disease was present in 17 patients (17\%), whereas mild to moderate mitral valve insufficiency was present in 58 patients $(58 \%)$ with no significant differences associated with the type of $\mathrm{AF}$ $(P=.53)$. The presence of mild coronary artery disease was noted on preoperative angiogram in 23 patients $(23 \%)$, with no significant difference observed $(P=.30)$.

All patients with AF who were referred for minimally invasive bipolar radiofrequency ablation had previously failed medical therapy or catheter ablation. Some referred patients were not considered suitable candidates if they had previous thoracic surgery leading to severe adhesions, had an enlarged left atrium $(>6.0 \mathrm{~cm})$, had evidence of thrombus in the left atrium, were not able to tolerate single lung ventilation, or were considered too high risk because of age or comorbidities.

\section{Definitions of Atrial Fibrillation Type}

Paroxysmal AF was defined in this study as AF less than 7 days in duration and self-terminated. Persistent $\mathrm{AF}$ was defined as $\mathrm{AF}$ greater than 7 days in duration or terminated by direct-current cardioversion or pharmacologically within 7 days. Permanent AF (recently changed to long-standing persistent) was defined as continuous AF lasting more than 1 year in duration.

\section{Surgical Technique}

General endotracheal anesthesia is delivered via a double-lumen endotracheal tube. A central line is placed, and a transesophageal echocardiogram is performed to determine the presence or absence of clot in the LA appendage. If thrombus is identified, the procedure is aborted.

A video-assisted bilateral mini-thoracotomy technique is used to electrically isolate the pulmonary veins. The patient is positioned in the left lateral decubitus position, and a small incision is made at the intersection of the anterior axillary line and the line at the level of the xyphoid process. A 5-mm trochar is inserted, and a 30-degree degree angled 5-mm videoscope is directed through this port. An incision is made at the midaxillary line over the fourth intercostal space, and the intercostal muscle and the pleura are divided. A soft tissue retractor (CardioVations, Edwards Lifesciences Corp, Irvine, Calif) is placed.

The pericardium is divided anterior to the phrenic nerve from the superior vena cava to the inferior vena cava. Retraction sutures are placed on the inferior cut edge of the pericardium and brought out through separate small stab incisions on the lateral chest wall. The oblique and transverse sinuses are entered using blunt dissection, and an articulating lighted tip dissector (Navigator, Medtronic Inc, Minneapolis, Minn) is placed through a separate port. The dissector is introduced through the oblique sinus and directed posterior to the right pulmonary veins. The tip of the dissector is then guided between the right superior pulmonary vein and the right main pulmonary artery. A Silastic guide is then attached to the tip of the dissector via a guide wire and brought back through the dissector port.

Saline-irrigated and nonirrigated bipolar radiofrequency clamps (BP2 or Gemini, Medtronic Inc; Atricure Inc, Cincinnati, $\mathrm{OH}$ ) are applied to the pulmonary veins to achieve transmural lesions. The generator used to deliver the radiofrequency energy has a digital graph that displays conductance of the tissue between the clamp. Once the tissue becomes electrically inert, according to the predefined limits, a visual and audible signal is produced, as well as to ensure electrical isolation of the pulmonary veins by both sensing and pacing technology.

With the pulmonary veins anatomically isolated, a pacing/sensing and ablation pen/probe (MAPS pen, Medtronic Inc or Atricure Bipolar Pen, Atricure Inc) is used to identify AGP and ablate them. The pen/probe is placed in pacing mode, and high-frequency pacing $(800-1000 \mathrm{bpm})$ is applied to the fat pad overlying the right atrium and along Waterston's groove. Atrioventricular nodal slowing, as defined by a greater than $50 \%$ increase in the $\mathrm{R}$-to-R interval, is marked as a site of autonomic ganglion clusters. These sites (Figure 1) are then ablated with the bipolar radiofrequency pen/probe.

The pen/probe is also used to document preablation electrical conductivity across the pulmonary veins by both pacing and sensing the superior and inferior pulmonary veins. Once the baseline electrical activity is recorded, the bipolar radiofrequency clamp is positioned around the pulmonary veins. The Silastic guide directs the lower jaw of the clamp posterior to the right pulmonary veins. Once the jaws of the clamp are correctly positioned around the antrum of the pulmonary veins and onto the left atrium, bipolar energy is delivered to the tissue. We routinely performed 3 ablations for each lesion. After the ablation, the pulmonary veins are again tested for electrical activity; the pen/probe is used to sense and pace across the pulmonary veins. The inability to pace across the pulmonary veins (exit block) and the absence of atrial electrical activity on the pulmonary veins during sensing (entrance block) determine whether the PV ablation line is complete, and therefore whether further ablations are necessary. The latter was necessary in less than $10 \%$ of patients. The evaluation of entrance block is especially important in the setting of patients with permanent $\mathrm{AF}$ in whom cardioversion may not always be successful intraoperatively. The clamp is removed, and a $24 \mathrm{~F}$ Blake drain is placed. The port sites are closed once the right lung is reinflated.

The patient is repositioned into the right lateral decubitus position, and the procedure is reproduced on the left side. Instead of the fourth interspace for the working port, the third interspace is entered. The pericardium is divided posterior to the phrenic nerve from the pulmonary artery superiorly to the inferior pulmonary veins. The ligament of Marshall is divided, and testing for autonomic ganglion occurs along the Marshall tract. Entrance and exit block testing are performed once ablation is complete.

The LA appendage is excised using a $60-\mathrm{mm}$ endoGIA stapler (Auto suture, United States Surgical Corporation, Norwalk, Conn). The stapler is inserted through the same port as the bipolar clamp. If at the end of the procedure the patient is in $\mathrm{AF}$ or atrial flutter, synchronized direct-current cardioversion is performed.

\section{Follow up}

The mean follow-up was $13.6 \pm 8.2$ months and $100 \%$ complete; all patients had at least a 24-hour Holter monitor after a blanking period of 3 months and then on a recommended yearly basis. Procedural success was defined as the absence of AF or atrial flutter on follow-up electrocardiograms and Holter monitor recordings after the blanking period. Informed consent for continuous follow-up monitoring had been given a priori by the patients as part of a standardized protocol for this minimally invasive ablative therapy. All protocols were reviewed and approved by the institutional health research ethics board.

\section{Statistical Analysis}

Baseline patient characteristics were compared between patients' groups via chi-square test and Fisher's exact test when frequency was less than 5. Continuous variables were assessed using the Student $t$ test when normally distributed and the Wilcoxon rank-sum test when data were skewed. Oneway analysis of variance tests, with Bonferroni's $t$ test for the means, were performed on continuous variables that were dependent on a classification.

The Kaplan-Meier method was used to assess time-related outcome (recurrence of AF). Predictors of recurrence of AF were identified using 
TABLE 1. Patient characteristics

\begin{tabular}{|c|c|c|c|c|c|}
\hline Characteristic & All $N=100(\%)$ & Paroxysmal N = $39(\%)$ & Persistent $N=29(\%)$ & Permanent $\mathbf{N}=32(\%)$ & $P$ \\
\hline Male & $70(70)$ & $25(64)$ & $22(76)$ & $23(72)$ & .56 \\
\hline Age $(y$, mean $\pm S D)$ & $65 \pm 11$ & $65 \pm 11$ & $66 \pm 11$ & $64 \pm 11$ & .85 \\
\hline Weight $(\mathrm{kg})$ & $94 \pm 18$ & $94 \pm 18$ & $92 \pm 15$ & $97 \pm 21$ & .48 \\
\hline AF duration (mo) & $59 \pm 46$ & $59 \pm 46$ & $110 \pm 106$ & $83 \pm 43$ & .03 \\
\hline Previous DCC (no./pt) & $2.4 \pm 2.1$ & $2.1 \pm 1.1$ & $2.5 \pm 2.3$ & $2.7 \pm 2.5$ & .43 \\
\hline NYHA class I-II & $21(21)$ & $2(2)$ & $7(7)$ & $12(12)$ & .02 \\
\hline Hypertension & $65(65)$ & $29(74)$ & $15(52)$ & $21(66)$ & .15 \\
\hline Hypercholesterolemia & $25(25)$ & $7(18)$ & $7(24)$ & $11(34)$ & .28 \\
\hline Diabetes & $11(11)$ & $6(15)$ & $2(7)$ & $3(9)$ & .51 \\
\hline Smoking & $50(50)$ & $13(33)$ & $14(48)$ & $23(72)$ & .005 \\
\hline COPD & $5(5)$ & $4(10)$ & $1(4)$ & 0 & .13 \\
\hline TIA & $4(4)$ & $2(5)$ & $1(4)$ & $1(3)$ & .89 \\
\hline CVA & $9(9)$ & $1(3)$ & $3(10)$ & $5(16)$ & .15 \\
\hline Family history of CAD & $52(52)$ & $15(39)$ & $13(45)$ & $24(75)$ & .006 \\
\hline Amiodarone & $22(22)$ & $9(23)$ & $7(24)$ & $6(19)$ & .86 \\
\hline Sotalol & $8(8)$ & $5(13)$ & $2(7)$ & $1(3)$ & .31 \\
\hline Flecainamide & $15(15)$ & $6(15)$ & $7(24)$ & $2(6)$ & .15 \\
\hline Beta-blockers & $58(58)$ & $23(59)$ & $17(59)$ & $18(56)$ & .97 \\
\hline $\mathrm{Ca}^{++}$channel blockers & $22(22)$ & $8(21)$ & $5(17)$ & $9(28)$ & .57 \\
\hline Digoxin & $21(21)$ & $6(15)$ & $5(17)$ & $10(31)$ & .22 \\
\hline Coumadin & $78(78)$ & $29(74)$ & $20(69)$ & $29(91)$ & .09 \\
\hline Aspirin & $19(19)$ & $6(15)$ & $10(34)$ & $3(9)$ & .03 \\
\hline \multicolumn{6}{|l|}{ Echocardiography } \\
\hline LA dimension $(\mathrm{cm})$ & $4.3 \pm 0.6$ & $4.1 \pm 0.5$ & $4.2 \pm 0.5$ & $4.6 \pm 0.6$ & .0003 \\
\hline $\mathrm{EF}(\%)$ & $55 \pm 8.5$ & $57 \pm 6$ & $56 \pm 11$ & $52 \pm 8$ & .04 \\
\hline $\mathrm{PAP}(\mathrm{mm} \mathrm{Hg})$ & $31 \pm 2.9$ & $31 \pm 1.0$ & $31 \pm 1.5$ & $32 \pm 4.9$ & .98 \\
\hline
\end{tabular}

$A F$, Atrial fibrillation; $D C C$, direct-current cardioversion; $N Y H A$, New York Heart Association; $C O P D$, chronic obstructive pulmonary disease; $T I A$, transient ischemic attack; $C V A$, cerebrovascular accident; $C A D$, coronary artery disease; $C a^{++}$, calcium; $L A$, left atrium; $E F$, ejection fraction; $P A P$, pulmonary artery pressure; $S D$, standard deviation.

logistic regression and semiparametric multivariable Cox proportional hazard modeling; variables screening ensured that an adequate $(n=10)$ number of events was associated with a potential risk factor, and that the scales of ordinal and continuous variables were calibrated with respect to outcome. Variable selection proceeded in a forward stepwise manner with a liberal entry criterion of $P$ less than .15 and a stay criterion of $P$ less than .1. All analyses were performed using the SAS statistical software (SAS v9.1; SAS, Cary, NC).

\section{RESULTS}

\section{Preoperative and Operative Characteristics}

Table 1 shows the preoperative characteristics of patients undergoing the minimally invasive ablation; of note is the significantly longer duration of $\mathrm{AF}$ in patients with $\mathrm{AF}$ qualified as persistent or permanent $(P=.03)$. These latter patients were also found to experience more dyspnea $(P=$ $.02)$, have a greater proportion of smokers $(P=.005)$, and have more significant LA enlargement $(P=.0003)$.

The mean operative time was $253 \pm 65$ minutes. Intraoperative direct-current cardioversion was performed at the conclusion of the surgical procedure in 14 patients $(36 \%)$ with paroxysmal AF, 12 patients $(41 \%)$ with persistent $\mathrm{AF}$, and 21 patients $(66 \%)$ with permanent $\mathrm{AF}(P=.03)$. The number of patients leaving the operating room in normal sinus rhythm (NSR) was as follows: 37 (95\%) with parox- ysmal AF, $25(86 \%)$ with persistent $\mathrm{AF}$, and $24(75 \%)$ with permanent $\mathrm{AF}(P=.05)$.

\section{Outcomes}

In this series of patients, no intraoperative conversions to sternotomy or standard thoracotomy were required. There was no 30-day in-hospital mortality. The rate of postoperative procedure-related complications was $14 \%$ and included the need for permanent pacemaker in 5 patients $(5 \%)$, postoperative hemothorax managed conservatively in 3 patients $(3 \%)$, and phrenic nerve injury in 3 patients $(3 \%)$. Pulmonary embolus occurred in 1 patient $(1 \%)$ and transient ischemic attack occurred in 1 patient $(1 \%)$ on postoperative day 2.

The mean hospital length of stay was $6.5 \pm 3.9$ days (range 2-35, median 5 days). At the time of discharge, $67 \mathrm{pa}$ tients $(67 \%)$ were in NSR, 27 patients $(27 \%)$ were in $\mathrm{AF}, 1$ patient $(1 \%)$ was in atrial flutter, and 5 patients $(5 \%)$ were in NSR via a permanent pacemaker; there was no difference observed in discharge rhythm between patients with paroxysmal, persistent, or permanent $\mathrm{AF}(P=.26)$. Medications at the time of discharge included amiodarone in 52 patients $(52 \%)$, sotalol in 11 patients $(11 \%)$, flecainamide in 19 patients $(19 \%)$, beta-blockers in 50 patients $(50 \%)$, calcium 

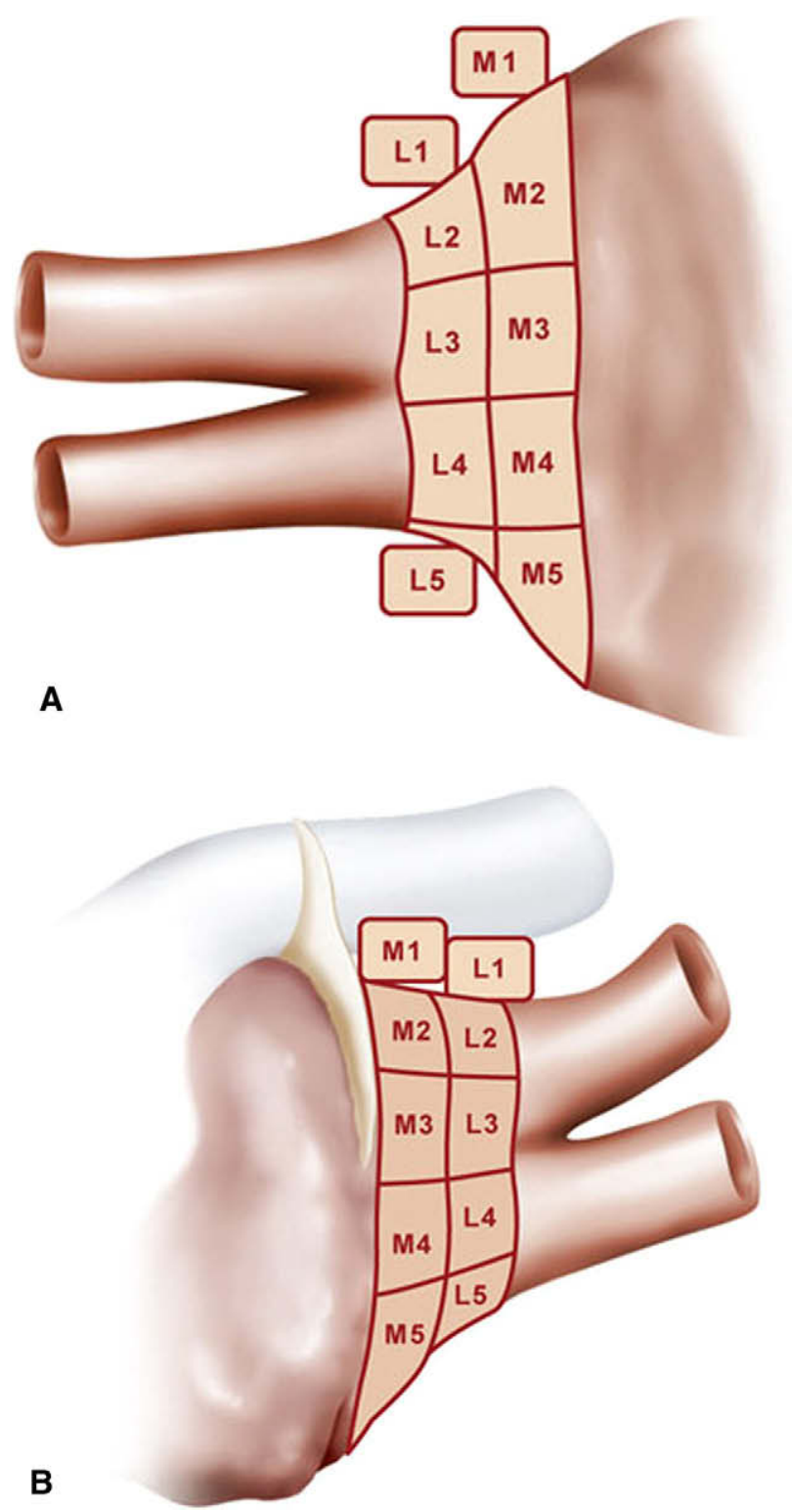

FIGURE 1. A, Right-sided AGP targeted for high-frequency stimulation and ablation. B, Left-sided AGP targeted for high-frequency stimulation and ablation.

channel blockers in 9 patients $(9 \%)$, digitalis in 14 patients $(14 \%)$, and warfarin in 86 patients $(86 \%)$.

At the time of follow-up, the proportion of patients in NSR was $87 \%$ overall. NSR was restored in $93 \%$ of patients with paroxysmal $\mathrm{AF}, 96 \%$ of patients with persistent $\mathrm{AF}$, and $71 \%$ of patients with permanent $\operatorname{AF}(P=.03)$ (Figure 2). Subjective reports of improvement in symptoms were observed in $87 \%$ of patients with paroxysmal AF, $100 \%$ of patients with persistent $\mathrm{AF}$, and $72 \%$ of patients with permanent $\operatorname{AF}(P=.007)$. Antiarrhythmic drugs and warfarin use were discontinued in $63 \%$ and $64 \%$ of patients, respectively, with no significant difference among AF type $(P=.27)$.

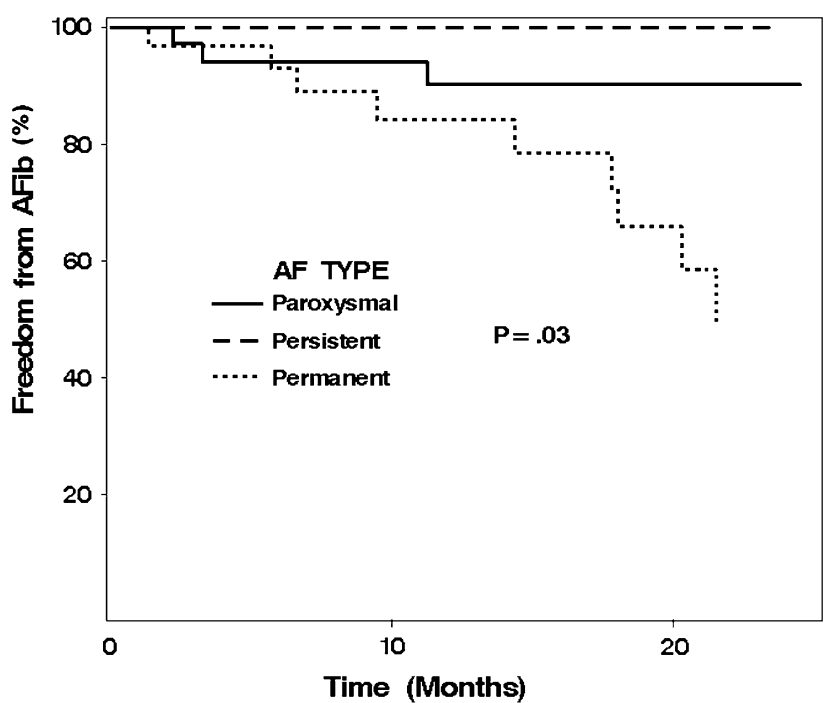

FIGURE 2. Freedom from AF in patients with paroxysmal, persistent, and permanent AF. $A F$, Atrial fibrillation.

Table 2 lists predictors of recurrence of AF with the strongest factor observed being the presence of permanent $\mathrm{AF}$ (hazard ratio 9.3; confidence interval, 2.2-38.5). The duration of AF was not found to be a significant predictor of recurrence of AF. There was a weak relationship observed between the presence of active autonomic ganglia on the left side superior pulmonary vein/ligament of Marshall areas and the recurrence of $\mathrm{AF}$ (hazard ratio 3.7; confidence interval, 1.1-13.2). By logistic regression, permanent AF, older age, and presence of AF at the time of discharge from the operating room were all strongly associated with recurrence of AF. There was no difference noted in the technology (irrigated vs nonirrigated) to create the pulmonary vein lesions $(P=.9)$. LA size was not found to be a predictor of failure to restore NSR.

\section{DISCUSSION}

During the last 20 years, surgeons and electrophysiologists have been making progress toward finding a cure for $\mathrm{AF}$ that offers patients an acceptable risk/benefit profile. The full cut-and-sew Maze procedure has delivered excellent results but has not been widely accepted as a stand-alone therapy ${ }^{17}$ Catheter-based techniques do offer a fast recovery when successful, but they have had modest success in restoring SR and still have measurable risk. ${ }^{10}$ Minimally invasive surgical techniques represent a compromise between these 2 approaches. Previous reports with alternate approaches have not delivered sustainable success. ${ }^{14}$ We believe that this approach offers satisfactory outcomes and brings us one step closer to therapy that offers our patients and referring colleagues an acceptable risk/benefit ratio.

The use of a bilateral thoracotomy approach for AF has been described for more than a decade. ${ }^{18}$ The reliability of bipolar radiofrequency ablation as an alternative to 
TABLE 2. Predictors of recurrence of atrial fibrillation

\begin{tabular}{lclc}
\hline \multicolumn{1}{c}{ Factor } & Coefficient \pm SD & $\boldsymbol{P}$ \\
\hline Proportional hazard & & & Hazard ratio $(\mathrm{CL})$ \\
Permanent AF & $2.2 \pm 0.72$ & .002 & $9.3(2.2-38.5)$ \\
AG_L1 & $1.3 \pm 0.66$ & .05 & $3.7(1.1-13.2)$ \\
Logistic regression & & & Odds ratio $(\mathrm{CL})$ \\
$\quad$ Permanent AF & $3.8 \pm 1.4$ & .007 & $45.5(2.8-75.1)$ \\
Older age & $0.08 \pm 0.04$ & .05 & $1.1(1.05-1.8)$ \\
Postoperative AF & $2.7 \pm 1.1$ & .01 & $14.7(1.9-60.5)$ \\
\hline$A F$, Atrial fibrillation; $A G_{-} L 1$, autonomic ganglion location 1; $S D$, standard deviation; \\
$C L$, confidence limit.
\end{tabular}

cut-and-sew techniques has led to an explosion of AF surgeries in patients undergoing cardiac surgery. ${ }^{19}$ However, its success after use on a beating heart with only the creation of pulmonary vein islands has been limited to single-institution case series. This multi-institutional report represents the largest experience to date and offers results from 3 surgeons.

Our early results suggest that the procedure is safe, with a complication rate lower than those reported with the classic Maze procedure. ${ }^{20}$ In addition, in early follow-up, the procedure appears effective, particularly for paroxysmal and persistent AF. The majority of patients are removed from medication and anticoagulation, and are symptomatically improved.

The complication rate and recovery time were still higher than we would hope for and do not represent a benign procedure. We believe that the benefit outweighs the risk and offers an improvement over the traditional Maze approach, but more data are needed before making this claim. We hope that with more experience, the complication rate will decrease and the advance of new technology will make this even less morbid.

We understand that the AGP play an integral role in the induction and maintenance of $\mathrm{AF}^{21} \mathrm{We}$ also understand that their destruction may only be a temporary phenomenon, ${ }^{22}$ and thus may not lead to long-term success. The role of this portion of the therapy needs to be more completely understood in this and all other approaches to AF that rely on the destruction of atrial tissue.

We know that the atrial appendage plays a role in stroke in patients with $\mathrm{AF}^{23}$ We think that its ligation will offer a stroke benefit and may be a major benefit of the Maze procedure. However, the amount of investigation in this area makes any thoughts on this issue premature. More investigation, including randomized studies that answer this question, are needed.

In this report, the mean LA dimension was close to normal and relatively homogenous; therefore, no correlation could be established between LA dimension and recurrence of AF. This may have been one of the main contributors to our encouraging early results. We do note, however, that patients with permanent AF have significantly larger LA dimensions than their paroxysmal and persistent counterparts.

\section{Limitations}

This is a multi-institution, nonrandomized, observational clinical study in which group differences and known confounders were controlled for in the multivariable analysis. Despite the adequate sample size and statistical adjustments applied, unmeasured and unknown confounders may have influenced the results. As those of other observational cohorts, the results of these analyses may not be generalizable to all patients who have undergone this procedure at other centers.

The follow-up, although complete, remains short. The long-term efficacy can only be known with additional follow-up. Furthermore, the use of at least a 24-hour Holter may be insufficient in the detection of recurrent tachyarrhythmias; perhaps the future availability of permanent loop recorders in this setting will allow to us to better ascertain the procedure's long-term success. Until this latter becomes standard of care, we are confident that our follow-up protocols remain superior to the phone call follow-up methodology of the initial experience with the Maze procedure. ${ }^{20}$ The bar must be raised if we are to continue to offer patients any intervention for AF, including medical therapy.

\section{Conclusions}

We believe that this approach to AF brings us closer to a procedure that is efficacious and offers a risk/benefit profile that will allow a benefit to patients with $\mathrm{AF}$. If the results from this study are sustained, this approach is already a viable therapeutic option for appropriate patients.

\section{References}

1. Miyasaka Y, Barnes ME, Gersh BJ, et al. Secular trends in incidence of atrial fibrillation in Olmsted County, Minnesota, 1980 to 2000, and implications on the projections for future prevalence. Circulation. 2006;114:119-25.

2. Roy H, Talajic M, Dorian P, et al. Canadian Trial of Atrial Fibrillation: amiodarone v. propafenone or sotalol for maintenance of sinus rhythm. $N$ Engl J Med. 2000;342:913

3. Corley SD, Epstein AE, DiMarco JP, et al. Relationships between sinus rhythm, treatment, and survival in the Atrial Fibrillation Follow-Up Investigation of Rhythm Management (AFFIRM) Study. Circulation. 2002;109:1509-13.

4. Roy D, Talajic M, Nattel S, et al. Late-breaking clinical trial abstracts from the American Heart Association's Scientific Sessions 2007. Circulation. 2007;116: 2627-33.

5. Kannel WB, Abbott RD, Savage DD, et al. Epidemiology features of chronic atrial fibrillation: the Framingham Study. N Engl J Med. 1982;306:1018-22.

6. Hylek EM, D'Antonio J, Evans-Molina C, et al. Translating the results of randomized trials into clinical practice. Stroke. 2006;37:1075-80.

7. Cox JL, Schuessler, D'Agostino HJ Jr, et al. The surgical treatment of atrial fibrillation. III. Development of a definitive surgical procedure. J Thorac Cardiovasc Surg. 1991;101:569-83.

8. Cox JL, Ad N, Palazzo T, et al. Impact of the Maze procedure on the stroke rate in patients with atrial fibrillation. J Thorac Cardiovasc Surg. 1999;118:833-40.

9. Stulak JM, Sundt TM, Dearani JA, et al. Ten-year experience with the Cox-Maze procedure for atrial fibrillation: how do we define success? Ann Thorac Surg. 2007;83:1319-25.

10. Cappato R, Calkins H, Chen SA, et al. Worldwide survey on the methods, efficacy, and safety of catheter ablation for human atrial fibrillation. Circulation. 2005;111:1100-5.

11. Gammie JS, Haddad M, Milford-Beland S, et al. Atrial fibrillation correction surgery: lessons from the Society of Thoracic Surgeons National Cardiac Database. Ann Thorac Surg. 2008;85:909-14. 
12. Pruitt JC, Lazzara RR, Dworkin G, et al. Totally endoscopic ablation of lone atrial fibrillation: initial clinical experience. Ann Thorac Surg. 2006;81:1325-31.

13. Ninet J, Roques X, Seitelberger R, et al. Surgical ablation of atrial fibrillation with off-pump, epicardial, high-density focused ultrasound: results of a multicenter trial. J Thorac Cardiovasc Surg. 2005;130:803-9.

14. Pruitt JC, Lazzara RR, Ebra G, et al. Minimally invasive surgical ablation of atrial fibrillation: the thoracoscopic box lesion approach. J Interv Card Electrophysiol. 2007;20:83-7.

15. Wolf RK, Schneeberger EW, Osterday R, et al. Video-assisted bilateral pulmonary vein isolation and left atrial appendage exclusion for atrial fibrillation. J Thorac Cardiovasc Surg. 2005;130:797-802.

16. Sagbas E, Akpinar B, Sanisoglu I, et al. Video-assisted bilateral epicardial pulmonary vein isolation for the treatment of lone atrial fibrillation. Ann Thorac Surg. 2007;83:1724-30.

17. McCarthy PM, Gillinov AM, Castle L, et al. The Cox-Maze procedure: the Cleveland Clinic experience. Semin Thorac Cardiovasc Surg. 2000;12:25-9.
18. Lee R, Takaski N, Schuessler RB, et al. The closed heart MAZE: a nonbypass surgical technique. Ann Thorac Surg. 1999;67:1692-702.

19. Gillinov AM, McCarthy PM, Blackstone EH, et al. Surgical ablation of atrial fibrillation with bipolar radiofrequency as the primary modality. $J$ Thorac Cardiovasc Surg. 2005;129:1322-9.

20. Prasad SM, Maniar HS, Camillo CJ, et al. The Cox maze III procedure for atrial fibrillation: long-term efficacy in patients undergoing lone versus concomitant procedures. J Thorac Cardiovasc Surg. 2003;126:1822-8.

21. Lemola K, Chartier D, Yeh YH, et al. Pulmonary vein region ablation in the experimental vagal atrial fibrillation role of pulmonary veins versus autonomic ganglia. Circulation. 2008;117:470-7.

22. Oh S, Zhang Y, Bibevski S, et al. Vagal denervation and atrial fibrillation inducibility: epicardial fat pad ablations does not have long term effects. Heart Rhythm. 2006;3:701-8.

23. Blackshear JL, Odell JA. Appendage obliteration to reduce stroke in cardiac surgical patients with atrial fibrillation. Ann Thorac Surg. 1996;61:755-9. 